\title{
Genes significantly associated with lineage II food isolates of Listeria monocytogenes
}

\author{
Cary Pirone-Davies ${ }^{1 *}$ (D), Yi Chen ${ }^{1}$, Arthur Pightling ${ }^{2}$, Gina Ryan ${ }^{1}$, Yu Wang ${ }^{2}$, Kuan Yao ${ }^{1}$, Maria Hoffmann \\ and Marc W. Allard ${ }^{1}$
}

\begin{abstract}
Background: Listeria monocytogenes is a widespread foodborne pathogen that can cause listeriosis, a potentially fatal infection. L. monocytogenes is subdivided into four phylogenetic lineages, with the highest incidence of listeriosis occurring within lineage I followed by lineage II. Strains of L. monocytogenes differ in their phenotypic characteristics, including virulence. However, the genetic bases for these observed differences are not well understood, and current efforts to monitor $L$. monocytogenes in food consider all strains to be equally virulent. We use a comparative genomics approach to identify genes and single nucleotide polymorphisms (SNPS) in 174 clinical and food isolates of L. monocytogenes that potentially contribute to virulence or the capacity to adapt to food environments.

Results: No SNPs are significantly associated with food or clinical isolates. No genes are significantly associated with food or clinical isolates from lineage I, but eight genes consisting of multiple homologues are associated with lineage II food isolates. These include three genes which encode hypothetical proteins, the cadmium resistance genes cadA and $c a d C$, the multi-drug resistance gene $e b r B$, a quaternary ammonium compound resistance gene qac, and a regulatory gene. All eight genes are plasmid-borne, and most closed L. monocytogenes plasmids carry at least five of the genes (24/27). In addition, plasmids are more frequently associated with lineage II food isolates than with lineage II clinical isolates.

Conclusions: We identify eight genes that are significantly associated with food isolates in lineage II. Interestingly, the eight genes are virtually absent in lineage II outbreak isolates, are composed of homologues which show a nonrandom distribution among lineage I serotypes, and the sequences are highly conserved across 27 closed Listeria plasmids. The functions of these genes should be explored further and will contribute to our understanding of how L. monocytogenes adapts to the host and food environments. Moreover, these genes may also be useful as markers for risk assessment models of either pathogenicity or the ability to proliferate in food and the food processing environment.
\end{abstract}

Keywords: Listeria, Comparative genomics, Virulence, Adaptation, Plasmid

\section{Background}

Listeria monocytogenes is a facultative intracellular pathogen that causes listeriosis, a predominantly foodborne disease with high case fatality and hospitalization rates (up to 19 and 94\%, respectively) [1]. Immunocompromised individuals including the elderly, infants, and

\footnotetext{
* Correspondence: cary.pirone@fda.hhs.gov

${ }^{1}$ Division of Microbiology, Office of Regulatory Science, Center for Food Safety and Applied Nutrition, U.S. Food and Drug Administration, College Park, MD, USA

Full list of author information is available at the end of the article
}

pregnant women, are at particularly high risk for contracting invasive listeriosis [2, 3].

Rates of $L$. monocytogenes infection have been difficult to control, in part, due to the widespread dissemination of $L$. monocytogenes in the natural environment. Outside of its animal hosts, L. monocytogenes survives as a saprotroph, colonizing diverse natural and urban settings. $L$. monocytogenes is also highly resilient to various stresses. It can survive refrigeration temperatures of $4{ }^{\circ} \mathrm{C}$ [4], salt concentrations as high as $13.9 \%$ and $\mathrm{pH}$ values as low as $4.1[5,6]$. This capacity for tolerating extreme conditions which inhibit the growth of most foodborne pathogens

(c) The Author(s). 2018 Open Access This article is distributed under the terms of the Creative Commons Attribution 4.0 International License (http://creativecommons.org/licenses/by/4.0/), which permits unrestricted use, distribution, and 
makes it of particular concern for the food industry. Currently, the U.S. enforces a zero tolerance policy for L. monocytogenes in ready to eat foods, such that if a detectable level of $L$. monocytogenes is present in a food (1 CFU L. monocytogenes per $25 \mathrm{~g}$ food), that food is deemed a hazard to health [7].

L. monocytogenes consists of four phylogenetic lineages [8-12] that vary in their ecological, evolutionary, and phenotypic characteristics, including virulence [11-15]. There is strong evidence that strains belonging to lineage I are on average more virulent than those from lineage II. Lineage I strains are more frequently associated with human clinical cases and are predominantly linked to outbreaks involving invasive disease [16]. Additionally, in vitro and animal studies indicate invasion and disease phenotypes are more frequent among lineage I strains compared to lineage II strains [17]. Furthermore, strains from lineage I are highly clonal [18], suggesting that genetic traits important for fitness within the host are under strong selection. Lineage II strains show higher rates of recombination than those from lineage I, which may contribute to an enhanced capacity to adapt to various ecological niches [18]. This increased genomic plasticity among lineage II strains supports the findings from several studies that show lineage II isolates are more frequently isolated from a broad diversity of sources, including foods, than lineage I strains $[19,20]$. Strains from lineages III and IV are rarely associated with human disease and are predominantly isolated from animal sources [21, 22].

L. monocytogenes is further categorized into 13 serotypes [23] and numerous clonal groups [24, 25] that exhibit heterogeneity in virulence both within and among lineages $[14,24,25]$. Lineage I serotypes $1 / 2 b, 4 b$, and lineage II serotype $1 / 2$ a are responsible for $>95 \%$ of human illness [26], while clonal complexes CC1 and CC6 are associated with the highest incidences of infection $[14,27]$.

Stress tolerance promotes survival in either the host or certain environmental niches and may indirectly influence virulence by increasing isolate abundance in the food supply and in turn, increase the chances of an isolate reaching and infecting a host. Some lineage II strains show an enhanced capacity to survive and grow under various stressors when compared to lineage I strains [13], and serotype $4 \mathrm{~b}$ isolates show enhanced survival after heat treatment following cold storage when compared to $1 / 2 \mathrm{a}$ isolates [28].

The genetic bases for differences in virulence and stress tolerance are not well understood, although many genes involved in these traits have been documented (for reviews on virulence genes, see [29-32], stress tolerance genes [33-35]). Many strains carrying a truncated internalin A (inlA) show reduced virulence [36, 37], but few genomic markers can reliably predict the capacity for virulence. Most recognized virulence genes (e.g., inlB, $\operatorname{prf} A$, and $\operatorname{sig} B$ ) are largely conserved across the species $[14,22,38]$, except the pathogenicity island LIPI-3, which is largely restricted to lineage I isolates [38], and the Listeria pathogenicity island 4 (LIPI-4), implicated in neural and placental infections [14], is present only in a subset of isolates [14,38]. Gene losses and truncations among several virulence genes have been observed and may account, in part, for attenuated virulence [14, 22, 38]. However, deletions and truncations are also observed in highly virulent strains, indicating that these mutations are not solely responsible for observed differences in virulence [14, 22, 38]. Comparative genomics studies in L. monocytogenes have focused on the association between virulence and clonal groups or sublineages. No large-scale study has examined the association between virulence or stress tolerance genes and isolation source, although data from cell culture and animal model studies indicate that clinical isolates are, on average, more virulent than food isolates [39-41]. Neither has there been a large-scale comparative study that has examined the association between single nucleotide polymorphisms (SNPs) and isolation source. SNPs resulting in a non-synonomous substitution may affect gene function and contribute to observed phenotypic differences among strains.

Thus, to assess genetic differences across the species, we compare the genomes of $174 \mathrm{~L}$. monocytogenes isolates to identify genes and SNPs that are non-randomly associated with either food or clinical isolates. Current risk assessment models of $L$. monocytogenes which predict the virulence of strains or the risk of certain foods would benefit greatly from additional genomic markers of virulence or enhanced survival in food [42]. These data will also enhance our understanding of how L. monocytogenes adapts to the host and food environments. This information is critical for reducing L. monocytogenes in the food supply and treating patients once they are infected.

\section{Methods \\ Isolate selection}

We selected 169 clinical, food, and food-environmental isolates from the Food and Drug Administration Center for Food Safety and Applied Nutrition (FDA-CFSAN) in-house collections and the Sequence Read Archive (SRA) database of NCBI, which are mainly isolates sequenced by the Center for Disease Control and Prevention (CDC) [43] (Additional file 1). Clinical isolates (total $n=79$, including reference isolates described below) were selected to represent the phylogenetic diversity present in L. monocytogenes including isolates from lineages I, II, and III. A phylogenetic tree containing all $L$. monocytogenes isolates $(n=2012)$ from 
the GenomeTrakr Project (Genbank Bioproject PRJNA215355, November, 2014) was constructed. Clinical isolates, defined as such by two attributes in the Genbank Biosample Database, attribute package $=$ clinical $/$ host-associated and isolation source = human, were selected from each major clade (data not shown). Metadata for all isolates including isolation source and date are presented in Additional file 1 . Food isolates $(n=95)$ include those from fresh produce, meat products (pork, poultry, beef), and dairy products (butter, milk, cheese), and industrial isolates consist of swabs of non-food contact surfaces from various meat production facilities. Food and environmental isolates were obtained from several randomly sampled United States Department of Agriculture (USDA) collection efforts, and no samples were epidemiologically linked to any cases of foodborne illness (P. Evans, USDA, personal communication). This set of food and meat-production facility isolates will be referred to broadly as food isolates in the remainder of the paper. Food isolates were also examined to ensure they represent the phylogenetic diversity of $L$. monocytogenes.

As outgroups for the phylogeny in Additional files 3 and 4, we included two previously sequenced $L$. innocua strains: ATCC33091 and FSL J1-023 [44]. L. monocytogenes reference genomes included EGD-e [45], EGD [46], and the high quality draft genomes SLCC2372, SLCC2540 [13], and FSL R2-503 [44]. Reference genome sequences were downloaded from Genbank [47]. A total of 176 isolates are described in Additional file 1, including two $L$. innocua isolates used only in the phylogenetic analysis. The remaining 174 isolates are included in the comparative analyses.

\section{Isolate culture and DNA extraction}

Each strain was plated onto Trypticase Soy Agar and incubated overnight at $37{ }^{\circ} \mathrm{C}$. Cells were then inoculated into Trypticase Soy Broth for DNA extraction using the DNeasy Blood and Tissue Kit (Qiagen, Valencia, CA, USA).

\section{Serotyping}

In silico serotyping was performed first by BLAST analysis of presence/absence of previously identified genomic serotype markers [48]. When atypical presence/ absence patterns were observed, in silico multilocus sequence typing (http://bigsdb.pasteur.fr/listeria/listeria.html) was used to help determine the serogroup.

\section{Determination of clonal complex}

ST and CC for these genomes were determined using the sequence query tool built in the Pasteur Listeria MLST database (http://bigsdb.pasteur.fr/listeria/listeria.html) on the basis of the definition by Ragon et al. [11].

\section{Sequencing and assembly}

Libraries were constructed for all FDA isolates listed in Table 1 using the Nextera XT Sample Preparation Kit (Illumina, Inc., San Diego, CA, USA), except for CFSAN028538 and CFSAN028542, which were previously constructed with the TruSeq DNA Library Preparation Kit (Illumina, Inc., San Diego, CA, USA). Paired-end DNA sequencing was performed on the Illumina MiSeq (Illumina, Inc., San Diego, CA, USA). Coverage was at least 50× for all isolates. All sequences were assembled using SPADES v.3.0.0 and contigs composed of less than 500 bp were excluded from the final assemblies. ORFs from each genome were annotated using the Prokka pipeline [49].

CFSAN022990 and CFSAN004330 were also sequenced on the PacBioRS II (Pacific Biosciences; Menlo Park, CA, USA) using standard protocols in order to identify the location of genes with high confidence. De-novo assembly was performed using the Hierarchical Genome Assembly Process (HGAP) with default parameters [50].

\section{Phylogeny reconstruction}

A maximum likelihood phylogeny of L. monocytogenes based on a 95\% majority SNP matrix was reconstructed using kSNP v2.1.2, with kmer size $=19$, as identified by the Kchooser script [51], and L. innocua as the outgroup.

\section{Comparative genomics}

Orthologous coding sequence clusters were identified bioinformatically with OrthoMCL [52] using default parameters and a Markov inflation parameter of 1.5 [44]. Coding sequences present in only a single genome, singletons, were identified using the orthomclSingletons perl script included in the OrthoMCL package. Singletons were excluded if they are less than 50 amino acids in length, and were added into existing clusters if they have a sequence similarity of $60 \%$ or higher, coverage greater than or equal to $80 \%$, and an e-value less than or equal to $10^{-5}$ when searched against sequences in each cluster using a protein BLAST. Orthologous clusters were used to determine the core and accessory genomes of L. monocytogenes. The core genome is defined as the set of genes present in all of the genomes analyzed, while the accessory genome is defined as the set of genes that were missing from at least one genome. A presence absence gene matrix was constructed from the OrthoMCL output.

\section{Occurrence of virulence and stress response genes across all genomes}

To determine the presence or absence of a previously described set of 125 virulence genes [22] we performed a nucleotide BLAST of each gene against each genome 
Table 1 Percent of clinical and food isolates containing significant genes in lineages I and II, and percent of outbreak isolates in lineages I and II containing significant genes

\begin{tabular}{|c|c|c|c|c|c|c|c|c|}
\hline \multicolumn{9}{|c|}{ Percent of isolates containing gene } \\
\hline Gene Name (PROKKA) & $\begin{array}{l}\text { lin I clinical } \\
(n=41)\end{array}$ & $\begin{array}{l}\operatorname{lin} \text { I food } \\
(n=34)\end{array}$ & $\begin{array}{l}\text { lin II clinical } \\
(n=35)\end{array}$ & $\begin{array}{l}\operatorname{lin} \| \text { food } \\
(n=60)\end{array}$ & $\begin{array}{l}\text { lin I clincal+ } \\
\text { food }(n=75)\end{array}$ & $\begin{array}{l}\text { lin II clinical+ } \\
\text { food }(n=95)\end{array}$ & $\begin{array}{l}\text { lin I outbreak } \\
\text { isolates }(n=27)\end{array}$ & $\begin{array}{l}\text { lin II outbreak } \\
\text { isolates }(n=22)\end{array}$ \\
\hline cadC & $30.95 \%$ & $45.45 \%$ & $20.00 \%$ & $73.33 \% \%^{*}$ & $37.33 \%$ & $53.68 \%$ & $48.15 \%$ & $13.64 \%$ \\
\hline $\operatorname{cadA}$ & $30.95 \%$ & $45.45 \%$ & $20.00 \%$ & $73.33 \% *$ & $37.33 \%$ & $53.68 \%$ & $48.15 \%$ & $13.64 \%$ \\
\hline hypothetical protein 1 & $21.43 \%$ & $33.33 \%$ & $14.29 \%$ & $66.67 \% *$ & $26.67 \%$ & $47.37 \%$ & $18.52 \%$ & $0.00 \%$ \\
\hline hypothetical protein 2 & $23.81 \%$ & $33.33 \%$ & $14.29 \%$ & $66.67 \% *$ & $28.00 \%$ & $47.37 \%$ & $18.52 \%$ & $22.73 \%$ \\
\hline hypothetical protein 3 & $21.43 \%$ & $30.30 \%$ & $8.57 \%$ & $63.33 \%^{*}$ & $25.33 \%$ & $43.16 \%$ & $22.22 \%$ & $13.64 \%$ \\
\hline ebrB & $2.38 \%$ & $21.21 \%$ & $8.57 \%$ & $53.33 \% *^{*}$ & $10.67 \%$ & $36.84 \%$ & $44.44 \%$ & $4.55 \%$ \\
\hline qac & $2.38 \%$ & $21.21 \%$ & $5.71 \%$ & $50.00 \% *$ & $10.67 \%$ & $33.68 \%$ & $44.44 \%$ & $4.55 \%$ \\
\hline regulatory gene & $2.38 \%$ & $21.21 \%$ & $8.57 \%$ & $51.67 \% *$ & $10.67 \%$ & $35.79 \%$ & $44.44 \%$ & $4.55 \%$ \\
\hline
\end{tabular}

Asterisks represent statistically significant results (statistics not performed in outbreak isolates, thus, these results are reported only as percentages)

assembly. Significant hits were defined as those with coverage of at least $80 \%$ and a percent identity greater than or equal to $80 \%$. All matches under this criteria also had an e-value less than or equal to $1 \mathrm{e}-100$.

We also defined a set of 60 genes important in stress responses to different environmental factors (heat, cold, oxidative, acid, and general) [33, 34, 53-61] (Additional file 2), and performed a nucleotide BLAST search of these genes against each genome assembly as above.

To determine the presence of truncations in virulence and adaptation genes, we translated sequences to amino acids, aligned with MUSCLE [62], and manually inspected for truncations. Truncations were defined as present if a sequence was missing at least ten amino acids from the end of the sequence as compared to the EGD-e reference sequence. Nucleotide sequence location within each contiguous sequence (contig) of the assembly was examined to ensure that the truncation did not occur due to its position at the ends of contigs.

\section{Determination of enriched genes in clinical, food, and environmental isolates}

We performed a Yates-corrected chi-square test with a Bonferroni correction for multiple testing on each orthologous gene cluster to determine whether members of a cluster are significantly associated $(p<.05)$ with either clinical or food isolates $(n=41$ lineage I clinical isolates, $n=34$ lineage I food isolates, $n=35$ lineage II clinical isolates, $n=60$ lineage II food isolates). We chose the conservative Bonferroni correction in order to reduce the probability of Type I errors in our analyses. We removed core genes from this analysis as well as genes that were present in less than $15 \%$ of all isolates.

\section{Determination of enriched SNPs in clinical, food, and environmental isolates}

Using the SNP matrix produced by kSNP, we used the Predict Phenotype from SNPs (PPFS2) (https://sourceforge.net/ projects/ppfs/files/) add on package to kSNP [63] to determine whether any SNPs could be used to correctly predict a clinical or food phenotype. PPFS2 identifies SNPs that are not randomly assigned to a phenotype based on the chi square probability. In brief, diagnostic SNPs were identified using the PPFS2 programs PickPhenotypeSubset, GetSNPprobs, and DiagnosticSNPs. GetSNPprobs is used to calculate the chi square probability that SNP alleles are distributed randomly with respect to phenotype for each SNP identified by kSNP. DiagnosticSNPs sorts the list of SNP probability values output by GetSNPprobs. Starting with the SNP with the lowest $p$-value, DiagnosticSNPs calculates the accuracy, positive predictive value (PPV), and negative predicted value (NPV) (see full reference [57] for details). SNPs are then sequentially added from the sorted list, and accuracy, PPV, and NPV are recalculated. This is repeated until the accuracy, PPV, and NPV values decline, at which point the SNPs used in the calculation are defined as diagnostic SNPs. We determined the diagnostic SNPs for lineage 1 , lineage 2 , and across all $L$. monocytogenes lineages.

\section{Results \\ Identification of virulence and stress tolerance gene clusters}

The core genome of 174 isolates consists of 2322 genes, the accessory genome 2381 genes, and the pan genome 4703 genes, which is in the order of prior core genome calculations for L. monocytogenes [22, 44]. Also similar to previous studies, we identified variations among the virulence genes of our dataset, although none are significantly associated with either clinical or food isolates [14, $22,38]$ (Additional file 3). Of the 125 virulence genes examined, 45 genes are either missing or truncated in some isolates (Additional file 3). The presence of pathogenicity island 1 (LIPI-1) is highly conserved among all L. monocytogenes isolates, with the exception of hemolysin LLO, which is truncated in two genomes. A five-gene stress survival islet (SSI_1) is missing in several 
lineage I and II isolates as previously observed [14, 22, 38]. The presence of pathogenicity island LIPI-3 (LIPI-3) is variable among lineage I isolates, and surprisingly, was also detected in a single isolate from lineage II. Although it has been detected in L. innocua [64], a complete LIPI-3 has not been previously detected in lineage II $L$. monocytogenes isolates, although a partial LIPI-3 is present in several lineage II isolates [38]. Some virulence loci occur more frequently among lineage I or lineage II isolates, suggesting that they may be more important for virulence in one or the other lineage. Lmo1082, previously found to be up-regulated in vivo during infection in EGD-e [65], occurs more frequently among lineage II isolates than lineage I isolates $(88 \%$ and $57 \%$, respectively). In contrast, Imo0320, an LPXTG surface protein required for entry into some mammalian cells [66], is present in higher frequency among lineage I food isolates than lineage II food isolates $(100 \%$ and $56 \%$, respectively). Other genes are missing from taxa in both lineages, including lmo0263 (inlH), lmo0478, and lmo2558, suggesting a less essential role in virulence for these loci than for those loci which are conserved across all taxa. Internalin A is truncated more frequently in food isolates than in clinical isolates as has been reported previously; truncations occurred in 36\% of all food isolates averaged across lineages I and II [67]. No other truncations in virulence genes were significantly associated with isolation source.

Stress response genes are highly conserved among all lineages and both isolation sources, even more so than the virulence genes (Additional file 4). A single significant stress tolerance gene, $\operatorname{cadA}$, important for cadmium resistance, is significantly associated with food isolates in lineage II. No truncations are associated with a single isolation source, but truncations in $\operatorname{lmo1589} \operatorname{argB}$, and lmo1433 are frequently present in lineage I isolates, but not lineage II.

\section{Analysis of gene clusters for significant association with isolation source}

We analyzed OrthoMCL gene clusters to search for additional genes that may be significantly associated with isolation source. Clusters containing core genes or genes present in less than $15 \%$ of all isolates were not considered. In lineage I no clusters were identified, but eight clusters are significantly associated with food isolates in lineage II out of 778 gene clusters analyzed (Table 1, Additional file 5, Additional file 6). The eight significant lineage II clusters contain genes encoding for three hypothetical proteins (referred to here as hypothetical protein genes $1-3$, ), as well as the cadmium resistance genes $\operatorname{cad} A$ and $\operatorname{cadC}$ ( $c a d A$ noted above), the multi-drug resistance gene $e b r B$, the qac gene involved in resistance to quaternary ammonium compounds, and the nucleoid occlusion factor $\operatorname{sim} A$ (sequences listed in Additional file 7). Seven of the eight significant gene clusters identified by OrthoMCL contain multiple homologous sequences; cadA and cadC clusters each consist of seven homologues, gene clusters encoding hypothetical proteins 1 and 2 each contain three homologues, the gene cluster encoding $e b r B$ and $q a c$ has four and three homologues, respectively, and the $\operatorname{sim} A$ gene cluster has two.

To assess the accuracy of the PROKKA annotations for the genes identified in lineage II clusters, we compared the sequences and their homologues to published gene sequences. Four cadmium resistance cassettes have been described in L. monocytogenes, each of which consists of a P-type ATPase, cadA, and its putative transcriptional regulator, cadC. Genes from the same cassette occur as a pair within a genome, e.g., cadA1 occurs with cadC1, cadA2 occurs with cadC2, etc.; and $\operatorname{cad} A C$ is used to designate the pair. In our data, the four $\operatorname{cad} A$ and $\operatorname{cad} C$ homologues share $100 \%$ nucleotide sequence similarity with cadA1-cadA4 from L. monocytogenes. The three additional homologues, referred to here as $c a d A$ and $c a d C$ (5-7), contain the same NCBI CDD domains as those found in cadA1-A4. The multidrug resistance gene $e b r B$ has not been characterized in Listeria, but the $e b r B$ homologues identified here contain the same domain as ebrB from Bacillus (Name: emrE; NCBI CDD Accession: COG:2076). All homologues in the cluster annotated as $q a c$ also contain this domain, as do published qacC sequences from Staphylococcus aureus. Sequences of homologues from the ebrB and qac clusters show $41-52 \%$ sequence identity at the amino acid level when compared against each other. In contrast, the sequences in the cluster annotated as $\operatorname{sim} A$ do not contain the same domains as $\operatorname{sim} A$ characterized from $E$. coli. Sequences in this study designated as $\operatorname{sim} A$ and $\operatorname{sim} A$ from $E$. coli both share the TetR_N superfamily domain, but our homologues contain the domain acrR (NCBI CDD Accession: COG1309) instead of the domain $\operatorname{sim} A$ (NCBI CDD Accession: PRK09480). The correct annotation for these sequences is unclear, but we assume that they have a regulatory role based on the presence of the acrR domain (DNA-binding transcriptional regulator, AcrR family). This cluster will therefore be referred to as the "regulatory gene cluster" in the remainder of the discussion.

The function of the three genes encoding hypothetical proteins is unknown. Using BLAST, we searched each sequence against the non-redundant (nr) database, but hits consist only of other genes encoding hypothetical proteins in L. monocytogenes plasmids. These results suggest that the genes may be plasmid-borne. There are 
no hits when searching against the COG and PFAM databases.

All eight gene clusters found significantly associated with lineage II food isolates are likely plasmid encoded, although some cadAC homologues may be found on other mobile elements. We performed a BLAST search of the most abundant homologue of each of the eight genes against the $\mathrm{nr}$ database, and found that the top hits for each sequence are closed plasmids (coverage of 99-100\%, percent identity 99-100\%). Furthermore, we performed a nucleotide BLAST of the genes against 27 publicly available closed Listeria plasmids from Genbank (October 2017) (Additional file 8). All but a single plasmid contain at least one homologue of $\operatorname{cadAC}$, and $25 / 27$ contain genes encoding hypothetical proteins 2 and 3 (sequence similarity greater than $60 \%$ and coverage of at least $80 \%$ ). The gene encoding hypothetical protein 1 is present in 15/27 plasmids, while $e b r B$, qac, and the regulatory gene are present in $8 / 27$. The conservation of the genes across diverse plasmids suggest they may confer a selective advantage. To verify the position of the eight genes in isolates of our dataset, we closed the genomes of CFSAN0022990 and CFSAN004330, two food isolates from this study, using PacBio technology. CFSAN022990 bears a single plasmid containing all eight significant genes ( $99 \%$ similar to reference genome L. monocytogenes $6179,90 \%$ coverage), as does CFSAN004330, albeit different homologues.

\section{Presence of eight genes among serotypes}

In lineage II, over half of serotype groups $1 / 2$ a plus $3 \mathrm{a}$ (referred to here as serotype group $1 / 2 \mathrm{a}$ ) and $1 / 2 \mathrm{c}$ plus $3 \mathrm{c}$ isolates (serotype group $1 / 2 \mathrm{c}$ ) contain at least two of the eight genes (41/77 and 10/15, respectively). In both serotypes, cadAC1 and cadAC2 predominate over other cadAC homologues, along with the primary homologues of genes encoding hypothetical proteins 1-3 when cadAC1 is present and the second homologue of these proteins when cadAC2 is present (Fig. 1, Additional file 6). In lineage I, the eight genes are present in (24/74) isolates able to be serotyped, and occur more frequently among serotypes $1 / 2 b, 3 b$, and 7 (serotype group $1 / 2 b$ ), than among $4 \mathrm{~b}, 4 \mathrm{~d}, 4 \mathrm{e}$ (serotype group $4 \mathrm{~b})$ isolates $(18 /$ 35 and 6/34, respectively), and none of the five $4 \mathrm{~b}$ variant (4bv) isolates contained any of the genes. The proportion of homologues of each of the eight genes present in $1 /$ $2 \mathrm{~b}$ isolates is similar to that of lineage II food isolates. In the few $4 \mathrm{~b}$ isolates containing any of the genes, the homologues of cadAC vary ( 2 cadAC1, 1 cadAC2, 3 cadAC4, and 1 cadAC6), and most isolates do not have homologues of any of the three hypothetical protein encoding genes, the qac genes, or the regulatory gene (Additional file 6). Please note, references to serotype groups include clinical and food isolates.

\section{Estimation of identified genes in outbreak isolates}

We determined how frequently the eight significant genes from lineage II occurred in a set of outbreak isolates from lineages I $(n=27)$ and II $(n=22)$ compiled from a recent publication and our internal database [27] (Additional file 9). Consistent with the low frequency of the eight genes in lineage II clinical isolates, only $4 / 22$ lineage II outbreak isolates contain any of the eight genes, and none contain the gene encoding hypothetical protein 1 (Table 1 ).

\section{Identification of SNPs}

No SNPs in any core genome gene are statistically significantly nonrandomly associated with food or clinical isolates in lineages I or II. Several SNPs are statistically significantly associated with food or clinical isolates in the accessory genome, but both SNP alleles of all SNPs are associated with a single isolation source, while the SNP is often absent from the genomes of the other isolation source. Thus, there are no informative alternative SNP nucleotide states. Furthermore, all SNPs occur either within or between genes identified in the gene cluster analyses, or just below the threshold of significance. Thus, we do not feel that these SNPs provide much novel information not already discussed elsewhere in the paper and thus they are not reported here.

\section{Discussion}

We identify eight genes that are significantly associated with food isolates across lineage II. This is the first comparative genomics study to identify genes that are significantly associated with diverse food isolates in $L$. monocytogenes, although one benzalkonium resistance gene was found to be associated with food-associated $L$. monocytogenes sublineages [38]. In lineage I, the eight genes are present in an overall lower frequency than in lineage II, and are not significantly associated with either food or clinical isolates. The distribution of homologues of the eight genes varies with serotype in lineage I. Homologues present in serotype group $1 / 2 \mathrm{~b}$ were similar to those of lineage II food isolates, while serotype group $4 \mathrm{~b}$ cadAC homologues were varied and isolates were often missing the three hypothetical protein encoding genes found nonrandomly associated with lineage II food isolates. Previous studies showed that cadAC1 occurs less frequently in $4 \mathrm{~b}$ isolates than in $1 / 2 \mathrm{~b}$ isolates [68], and cadAC4 is present in a higher proportion of $4 \mathrm{~b}$ isolates than other serotypes [69].

Given the high degree to which the presence of these eight genes is conserved among phylogenetically diverse food isolates in lineage II, it is likely that the genes play an important role in the survival and proliferation of $L$. monocytogenes in the food environment. Understanding the mechanisms that underlie these processes is critical 


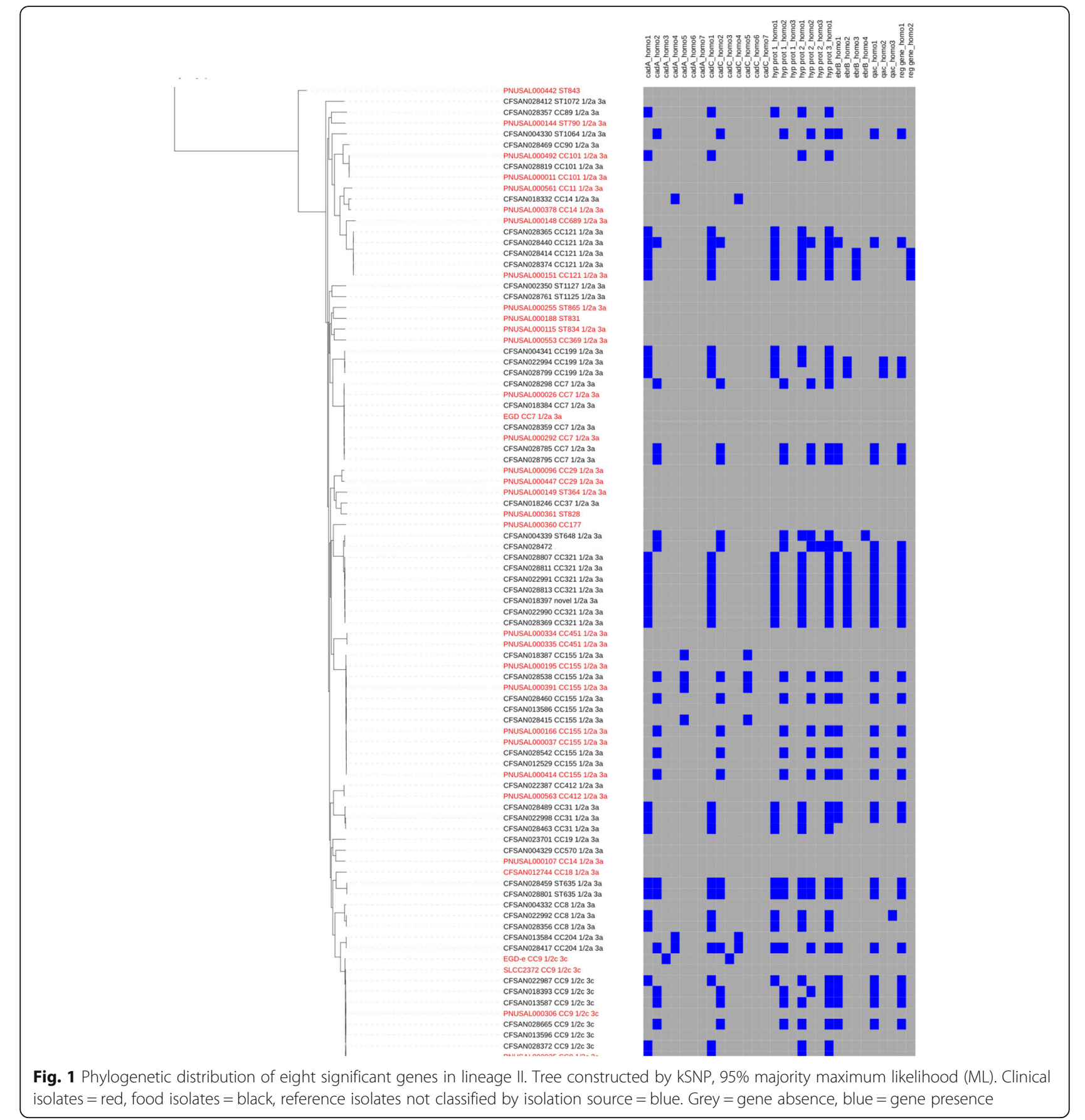

for the development of management practices to reduce L. monocytogenes in the food supply. In addition, functional information may be useful in predicting the risk associated with certain foods. This is particularly important as recent outbreaks have occurred in foods previously categorized as low risk such as ice cream and produce [42].

Collectively, little is known about the function of the eight genes. The heavy metal transporting efflux pump $\operatorname{cad} A$ and its putative repressor $\operatorname{cad} C$ expel toxic cadmium from the cell [70], while multidrug resistance genes such as $e b r B$ and genes encoding for resistance to quaternary ammonium compounds commonly used as industrial cleaners also reduce the level of toxic compounds in the cell [71]. Thus, the presence of cadAC, $e b r B$, and $q a c$ in lineage II food isolates likely promotes survival in food and in the food-processing environment by mitigating cell exposure to harmful chemicals. No 
information is available regarding the function of the three genes encoding hypothetical proteins or the acrR domain containing regulatory gene.

It is less clear why the eight loci are found in low frequencies among lineage II clinical isolates. Antibiotic resistance is often associated with infection [72]. Some of the top-infecting $L$. monocytogenes serotypes $1 / 2 \mathrm{a}, 1 / 2 \mathrm{~b}$, and $4 \mathrm{~b}[68]$ and outbreak isolates, as well as $1 / 2 \mathrm{~b}$ isolates in this study, contain cadA1 and cadA2. Furthermore, as a facultative pathogen, $L$. monocytogenes spends part of its life cycle in the environment before infecting a host, thus clinical isolates should also benefit from the genes. It is possible that the genes and/or mobile elements on which they reside confer a selective disadvantage within the host that outweighs any advantage gained while in the environment, or perhaps clinical isolates are transient in the environment, with few opportunities to acquire foreign DNA from other environmental isolates. The functions of the genes in lineages I and II may also vary against the different genetic background of the two lineages.

The function of cadAC beyond cadmium resistance has not been well explored, and studies do suggest that some cadAC homologues have a role in virulence. The expression of the negative regulator cadC3 in EGD-e (lmo1102) is up regulated during in vivo infection and was determined to be essential for virulence [65]. However, a recent study showed that the inactivation of cadA4 enhances virulence in a Galleria insect model [69], and in vivo, CadC3 is required for L. monocytogenes infection [73].

Given the presence of the eight genes on mobile elements, particularly plasmids, we were interested in testing whether plasmids are more frequent among food isolates than among clinical isolates in lineage II. To do this, we performed a nucleotide BLAST search of 27 closed $L$. monocytogenes plasmids against our genomes. Genomes of only $6 / 36$ clinical isolates have sequences similar to the plasmids, compared to $38 / 60$ food isolates (sequence similarity greater than $60 \%$ and plasmid coverage of at least $20 \%$ ). Correlations between plasmid presence and serotype have been noted previously in L. monocytogenes. In a group of 173 isolates collected in France, food and environmental isolates from serogroups 1 and 4 harbored plasmids more frequently than clinical isolates [74, 75]. In a collection of 322 UK isolates, plasmids were more frequently identified in serotype $1 / 2 \mathrm{a}$ food isolates than in clinical isolates, whereas the converse was true for serotype $1 / 2$ c [75]. Mobile genetic elements (MGE) such as plasmids expand the coding potential of the accessory genome, and increase bacterial fitness under some conditions. Thus, plasmids in food isolates likely provide a source of novel genetic information to adapt to diverse conditions encountered in food environments.
Although no statistically significant SNPs are reported here, additional studies which examine polymorphisms in groups of clinical and food isolates may yield insights into the mechanisms of adaptation or genetic markers to differentiate the two groups. For example, comparing the occurrence of SNPs among each core and accessory gene may identify genes with a higher frequency of mutation in either clinical or food isolates which could reflect the effect of different selection pressures on the two groups [76]. Assessing polymorphism differences between multiple pairs of genetically similar clinical and food isolates may identify markers to distinguish these isolates, as was demonstrated in a recent study which identified several SNPs which could differentiate adherent-invasive E. coli (AIEC) from non-AIEC strains [77]. In addition, other nonrandomly distributed polymorphisms such as insertions and deletions (indels) may be present in core genes and should be assessed in future studies utilizing reference-based methods.

\section{Conclusions}

Eight genes are significantly associated with food isolates in lineage II. Intriguingly, the eight genes are virtually absent in outbreak isolates from lineage II, are composed of homologues which show a unique distribution among serotypes in lineage I, and are highly conserved across 27 closed L. monocytogenes plasmids. Plasmids are also more frequently associated with lineage II food isolates than with clinical isolates. The role of the eight genes and plasmids in the survival and proliferation of $L$. monocytogenes in food and in the food-processing environment should be explored. This information is critical if we wish to control populations of $L$. monocytogenes in the food supply, particularly in light of the fact that recent $L$. monocytogenes outbreaks have occurred in foods that have historically been considered to be low risk. Future studies should assess these observations on a larger scale and explore the utility of the eight genes and their homologs in risk assessment models.

\section{Additional files}

\footnotetext{
Additional file 1: List of 176 isolates included in this study and the associated metadata. (XLSX $23 \mathrm{~kb}$ )

Additional file 2: List of stress tolerance genes included in this study. (XLSX $33 \mathrm{~kb}$ )

Additional file 3: Phylogenetic distribution of 45/125 virulence genes across $L$. monocytogenes (genes which are conserved are not shown). Blue $=$ present, yellow $=$ absent, gray $=$ truncated. Clinical isolates $=$ red, food isolates $=$ black, reference isolates not classified by isolation source = blue. (TIF $1165 \mathrm{~kb})$

Additional file 4: Phylogenetic distribution of 7/65 stress tolerance genes which vary across L. monocytogenes, (conserved genes not shown). Green $=$ present, purple $=$ absent, dark purple $=$ truncated. Clinical isolates $=$ red , food isolates $=$ black, reference isolates not classified by isolation source $=$ blue. $($ TIF $1104 \mathrm{~kb})$
} 
Additional file 5: Chi-Square test for significant gene clusters in lineage II. (XLSX $49 \mathrm{~kb})$

Additional file 6: Presence-absence matrix of eight genes across isolates in this study. Serotype, CC or ST, and the presence of a plasmid is indicated for each genome. (XLSX $37 \mathrm{~kb}$ )

Additional file 7: List of eight significant genes, their homologues, and sequence accessions. (XLSX $18 \mathrm{~kb}$ )

Additional file 8: List of plasmids downloaded from Genbank. (XLSX 9 $\mathrm{kb})$

Additional file 9: List of outbreak isolates. (XLSX $11 \mathrm{~kb})$

\section{Abbreviations}

CDC: Center for Disease Control and Prevention; contig: Contiguous sequence; FDA-CFSAN: Food and Drug Administration Center for Food Safety and Applied Nutrition; HGAP: Hierarchical Genome Assembly Process; LIPI-3: Pathogenicity island LIPI-3; ML: Maximum likelihood; NCBI: National Center for Biotechnology Information; NPV: Negative predicted value; PPFS2: Predict Phenotype from SNPs; PPV: Positive predictive value; SNP: Single nucleotide polymorphism; SRA: Sequence Read Archive; tRNA: Transfer RNA; USDA: United States Department of Agriculture; WGS: Whole Genome Sequencing

\section{Acknowledgements}

We thank Peter Evans for information regarding the metadata of the strain collections, and Heather Carleton and Steven Stroika for the sequencing and uploading of many clinical isolates to NCBI, as well as Cheryl Tarr and Zuzana Kucerova for sharing isolates from CDC. We also thank Justin Payne for his assistance with linux-related questions.

\section{Funding}

We thank the Research Fellowship Program for the Center for Food Safety and Applied Nutrition, administered by the Oak Ridge Associated Universities for funding. The funding body had no role in the design of the study and collection, analysis, and interpretation of data and in writing the manuscript.

\section{Availability of data and materials}

The datasets supporting the conclusions of this article are available in the Sequence Read Archive (SRA) of the National Center for Biotechnology Information (NCBI) (https://www.ncbi.nlm.nih.gov/sra), and the Whole Genome Sequencing (WGS) archive of NCBI (https://www.ncbi.nlm.nih.gov/ genbank/wgs/). Accessions are listed under Additional file 1.

\section{Authors' contributions}

CPD and MWA designed the study, analysis, and methods. CPD performed most of the analyses, with assistance from YW and AP. YC performed the serotyping and $\mathrm{MH}$ and $\mathrm{KY}$ closed genomes using pacbio data. CPD wrote the manuscript with assistance from GR and YC. All authors read and approved the final manuscript.

\section{Ethics approval and consent to participate}

Not applicable. All clinical isolates were obtained with IRB approval through agencies outside of the FDA. By the time samples are acquired by the FDA there is no metadata regarding patient identification associated with the samples.

\section{Consent for publication}

Not applicable.

\section{Competing interests}

The authors declare no competing of interest.

\section{Publisher's Note}

Springer Nature remains neutral with regard to jurisdictional claims in published maps and institutional affiliations.

\section{Author details}

'Division of Microbiology, Office of Regulatory Science, Center for Food Safety and Applied Nutrition, U.S. Food and Drug Administration, College
Park, MD, USA. ²Office of Analytics and Outreach, Center for Food Safety and Applied Nutrition, U.S. Food and Drug Administration, College Park, MD, USA

Received: 24 January 2018 Accepted: 12 September 2018

Published online: 25 September 2018

\section{References}

1. Scallan E, Hoekstra RM, Angulo FJ, Tauxe RV, Widdowson M-A, Roy SL, Jones JL, Griffin PM. Foodborne illness acquired in the United States-major pathogens. Emerg Infect Dis. 2011;17:7-15.

2. Mead PS, Slutsker L, Dietz V, McCaig LF, Bresee JS, Shapiro C, Griffin PM, Tauxe RV. Food-related deaths and illness in the United States. Emerg Infact Dis. 1999:5:607-25.

3. Allberger F, Wagner M. Listeriosis: a resurgent foodborne infection. Clin Microbiol Infect. 2010;6:16-23.

4. Greenwood MH, Roberts D, Burden P. The occurrence of Listeria species in milk and dairy products: a national survey in England and Wales. Int J Food Microbiol. 1991;12:197-206.

5. Shabala L, Lee SH, Cannesson P, Ross T. Acid and NaCl limits to growth of Listeria monocytogenes and influence of sequence of inimical acid and $\mathrm{NaCl}$ levels on inactivation kinetics. J Food Prot. 2008;71:1169-77.

6. Bergholz TM, den Bakker HC, Fortes ED, Boor KJ, Wiedmann M. Salt stress phenotypes in Listeria monocytogenes vary by genetic lineage and temperature. Foodborne Pathog Dis. 2010:7:1537-49.

7. Shank FR, Elliot EL, Wachsmuth IK, Losikoff ME. US position on Listeria monocytogenes in foods. Food Control. 1997:7:229-34.

8. Rasmussen OF, Skouboe P, Dons L. Listeria monocytogenes exists in at least three evolutionary lines: evidence from flagellin, invasive associated protein and listeriolysin 0 genes. Microbiology. 1995;141:2053-61.

9. Piffaretti JC, Kressebuch H, Aeschbacher M, Bille J, Bannerman E, Musser JM, Selander RK, Rocourt J. Genetic characterization of clones of the bacterium Listeria monocytogenes causing epidemic disease. Proc Natl Acad Sci U S A. 1989:86:3818-22.

10. Ward TJ, Ducey TF, Usgaard T, Dunn KA, Bielawski JP. Multilocus genotyping assays for single nucleotide polymorphism-based subtyping of Listeria monocytogenes isolates. Appl Environ Microbiol. 2008;74:7629-42.

11. Ragon M, Wirth T, Hollandt F, Lavenir R, Lecuit M, Le Monnier A, Brisse S. A new perspective on Listeria monocytogenes evolution. PLoS Pathog. 2008;4: e1000146.

12. Hof $H$, Rocourt J. Is any strain of Listeria monocytogenes detected in food a health risk? Int J Food Microbiol. 1992;16:173-82.

13. Orsi $\mathrm{RH}$, den Bakker HC, Wiedmann M. Listeria monocytogenes lineages: genomics, evolution, ecology, and phenotypic characteristics. Int J Med Microbiol. 2011:301:79-96.

14. Maury MM, Tsai YH, Charlier C, Touchon M, Chenal-Francisque V, Leclercq A Criscuolo A, Gaultier C, Roussel S, Brisabois A, et al. Uncovering Listeria monocytogenes hypervirulence by harnessing its biodiversity. Nat Genet. 2016;48:308-13.

15. den Bakker HC, Fortes ED, Wiedmann M. Multilocus sequence typing of outbreak-associated Listeria monocytogenes isolates to identify epidemic clones. Foodborne Pathog Dis. 2010;7:257-65

16. Lomonaco S, Nucera D, Filipello V. The evolution and epidemiology of Listeria monocytogenes in Europe and the United States. Infect Genet Evol. 2015:35:172-83.

17. Nightingale KK, Windham K, Martin KE, Yeung M, Wiedmann M. Select Listeria monocytogenes subtypes commonly found in foods carry distinct nonsense mutations in inlA, leading to expression of truncated and secreted internalin a, and are associated with a reduced invasion phenotype for human intestinal epithelial cells. Appl Environ Microbiol. 2005;71:8764-72.

18. den Bakker HC, Didelot X, Fortes ED, Nightingale KK, Wiedmann M. Lineage specific recombination rates and microevolution in Listeria monocytogenes. BMC Evol Biol. 2008:8:277.

19. Ward TJ, Gorski L, Borucki MK, Mandrell RE, Hutchins J, Pupedis K Intraspecific phylogeny and lineage group identification based on the prfA virulence gene cluster of Listeria monocytogenes. J Bacteriol. 2004;186:49945002

20. Gray MJ, Zadoks RN, Fortes ED, Dogan B, Cai S, Chen Y, Scott VN, Gombas DE, Boor KJ, Wiedmann M. Listeria monocytogenes isolates from foods and humans form distinct but overlapping populations. Appl Environ Microbiol. 2004;70:5833-41. 
21. Jeffers GT, Bruce JL, McDonough PL, Scarlett J, Boor KJ, Wiedmann M. Comparative genetic characterization of Listeria monocytogenes isolates from human and animal listeriosis cases. Microbiology. 2001;147:1095-104.

22. Kuenne C, Billion A, Mraheil MA, Strittmatter A, Daniel R, Goesmann A, Barbuddhe S, Hain T, Chakraborty T. Reassessment of the Listeria monocytogenes pan-genome reveals dynamic integration hotspots and mobile genetic elements as major components of the accessory genome. BMC Genomics. 2013;14:47.

23. Seeliger $\mathrm{H}$, Höhne K. Chapter II serotyping of Listeria monocytogenes and related species. Methods Microbiol. 1979;13:31-49.

24. Cantinelli T, Chenal-Francisque V, Diancourt L, Frezal L, Leclercq A, Wirth T, Lecuit M, Brisse S. "Epidemic clones" of Listeria monocytogenes are widespread and ancient clonal groups. J Clin Microbiol. 2011;51:3770-9.

25. Haase JK, Didelot X, Lecuit M, Korkeala H, Group LmMS, Achtman M. The ubiquitous nature of Listeria monocytogenes clones: a large-scale multilocus sequence typing study. Environ Microbiol. 2014;16:405-16.

26. Kathariou S. Listeria monocytogenes virulence and pathogenicity, a food safety perspective. J Food Prot. 2002;65:1811-29.

27. Chen Y, Gonzalez-Escalona N, Hammack TS, Allard MW, Strain EA, Brown EW. Core genome multilocus sequence typing for identification of globally distributed clonal groups and differentiation of outbreak strains of Listeria monocytogenes. Appl Environ Microbiol. 2016;82:6258-72.

28. Buncic S, Avery SM, Rocourt J, Dimitrijevic M. Can food-related environmental factors induce different behaviour in two key serovars, $4 \mathrm{~b}$ and 1/2a, of Listeria monocytogenes? Int J Food Microbiol. 2001;65:201-12.

29. Vazquez-Boland JA, Kuhn M, Berche P, Chakraborty T, Dominguez-Bernal G, Goebel W, Gonzalez-Zorn B, Wehland J, Kreft J. Listeria pathogenesis and molecular virulence determinants. Clin Microbiol Rev. 2001;14:584-640.

30. Hain T, Chatterjee SS, Ghai R, Kuenne CT, Billion A, Steinweg C, Domann E, Karst U, Jansch L, Wehland J, et al. Pathogenomics of Listeria spp. Int J Med Microbiol. 2007;297:541-57.

31. Camejo A, Carvalho F, Reis O, Leitao E, Sousa S, Cabanes D. The arsenal of virulence factors deployed by Listeria monocytogenes to promote its cell infection cycle. Virulence. 2011;2:379-94.

32. Cossart P. Illuminating the landscape of host-pathogen interactions with the bacterium Listeria monocytogenes. Proc Natl Acad Sci U S A. 2011;108: 19484-91.

33. Chaturongakul S, Raengpradub S, Wiedmann M, Boor K. Modulation of stress and virulence in Listeria monocytogenes. Trends Microbiol. 2008;16: 388-96.

34. Hill C, Cottera PD, Leatora RD, Gahan CGM. Bacterial stress response in Listeria monocytogenes: jumping the hurdles imposed by minimal processing. Int Dairy J. 2002;12:273-83.

35. Oliver HF, Orsi RH, Ponnala L, Keich U, Wang W, Sun Q, Cartinhour SW, Filiatrault MJ, Wiedmann M, Boor KJ. Deep RNA sequencing of $L$. monocytogenes reveals overlapping and extensive stationary phase and sigma B-dependent transcriptomes, including multiple highly transcribed noncoding RNAs. BMC Genomics. 2009;10:641.

36. Chen $Y$, Ross WH, Whiting RC, Van Stelten A, Nightingale KK, Wiedmann M, Scott VN. Variation in Listeria monocytogenes dose responses in relation to subtypes encoding a full-length or truncated internalin a. Appl Environ Microbiol. 2011;77:1171-80.

37. Nightingale KK, Ivy RA, Ho AJ, Fortes ED, Njaa BL, Peters RM, Wiedmann M inlA premature stop codons are common among Listeria monocytogenes isolates from foods and yield virulence-attenuated strains that confer protection against fully virulent strains. Appl Environ Microbiol. 2008;74: 6570-83.

38. Moura A, Criscuolo A, Pouseele H, Maury MM, Leclercq A, Tarr C, Bjorkman JT, Dallman T, Reimer A, Enouf V, et al. Whole genome-based population biology and epidemiological surveillance of Listeria monocytogenes. Nat Microbiol. 2016;2:16185.

39. Norrung $B$, Andersen JK. Variations in virulence between different electrophoretic types of Listeria monocytogenes. Lett Appl Microbiol. 2000; 30:228-32.

40. Norton DM, Scarlett JM, Horton K, Sue D, Thimothe J, Boor KJ, Wiedmann M. Characterization and pathogenic potential of Listeria monocytogenes isolates from the smoked fish industry. Appl Environ Microbiol. 2001;67:646-53.

41. Rakic Martinez M, Wiedmann M, Ferguson M, Datta AR. Assessment of Listeria monocytogenes virulence in the galleria mellonella insect larvae model. PLoS One. 2017;12:e0184557.
42. Buchanan RL, Gorris LGM, Hayman MM, Jackson TC, Whiting RC. A review of Listeria monocytogenes: an update on outbreaks, virulence, dose-response, ecology, and risk assessments. Food Control. 2017;75:1-13.

43. Leinonen $\mathrm{R}$, Sugawara $\mathrm{H}$, Shumway $\mathrm{M}$. on behalf of the international nucleotide sequence database $C$ : the sequence read archive. Nucleic Acids Res. 2011;39:D19-21.

44. den Bakker HC, Desjardins CA, Griggs AD, Peters JE, Zeng Q, Young SK, Kodira CD, Yandava C, Hepburn TA, Haas BJ, et al. Evolutionary dynamics of the accessory genome of Listeria monocytogenes. PLoS One. 2013;8:e67511.

45. Glaser P, Frangeul L, Buchrieser C, Rusniok C, Amend A, Baquero F, Berche P, Bloecker $\mathrm{H}$, Brandt $\mathrm{P}$, Chakraborty $\mathrm{T}$, et al. Comparative genomics of Listeria species. Science. 2001;294:849-52.

46. Becavin C, Bouchier C, Lechat P, Archambaud C, Creno S, Gouin E, Wu Z, Kuhbacher A, Brisse S, Pucciarelli MG, et al. Comparison of widely used Listeria monocytogenes strains EGD, 10403S, and EGD-e highlights genomic variations underlying differences in pathogenicity. MBio. 2014;5:e00969-14.

47. Clark K, Karsch-Mizrachi I, Lipman DJ, Ostell J, Sayers EW. GenBank. Nucleic Acids Res. 2016;44:D67-72.

48. Doumith M, Buchrieser C, Glaser P, Jacquet C, Martin P. Differentiation of the major Listeria monocytogenes serovars by multiplex PCR. J Clin Microbiol. 2004:42:3819-22.

49. Seemann T. Prokka: rapid prokaryotic genome annotation. Bioinformatics. 2014;30:2068-9.

50. Chin CS, Alexander DH, Marks P, Klammer AA, Drake J, Heiner C, Clum A, Copeland A, Huddleston J, Eichler EE, et al. Nonhybrid, finished microbial genome assemblies from long-read SMRT sequencing data. Nat Methods. 2013;10:563-9.

51. Gardner SN, Hall BG. When whole-genome alignments just won't work: KSNP V2 software for alignment-free SNP discovery and phylogenetics of hundreds of microbial genomes. PLoS One. 2013:8:e81760.

52. Li L, Stoeckert CJ Jr, Roos DS. OrthoMCL: identification of ortholog groups for eukaryotic genomes. Genome Res. 2003;13:2178-89.

53. Begley M, Hill C. Stress adaptation in foodborne pathogens. Annu Rev Food Sci Technol. 2015;6:191-210.

54. Fraser KR, Sue D, Wiedmann M, Boor K, O'Byrne CP. Role of B in regulating the compatible solute uptake systems of Listeria monocytogenes: osmotic induction of opuC is B dependent. Appl Environ Microbiol. 2003;69:2015-22.

55. Borezee E, Pellegrini E, Berche P. OppA of Listeria monocytogenes, an oligopeptide-binding protein required for bacterial growth at low temperature and involved in intracellular survival. Infect Immun. 2000;68: 7069-77.

56. Gahan CG, Hill C. Listeria monocytogenes: survival and adaptation in the gastrointestinal tract. Front Cell Infect Microbiol. 2014;4:9.

57. Seifart Gomes C, Izar B, Pazan F, Mohamed W, Mraheil MA, Mukherjee K, Billion A, Aharonowitz Y, Chakraborty T, Hain T. Universal stress proteins are important for oxidative and acid stress resistance and growth of Listeria monocytogenes EGD-e in vitro and in vivo. PLoS One. 2011;6:e24965.

58. Okada Y, Okada N, Makino S, Asakura H, Yamamoto S, Igimi S. The sigma factor RpoN (sigma54) is involved in osmotolerance in Listeria monocytogenes. FEMS Microbiol Lett. 2006;263:54-60.

59. Raimann E, Schmid B, Stephan R, Tasara T. The alternative sigma factor sigma $(L)$ of $L$. monocytogenes promotes growth under diverse environmental stresses. Foodborne Pathog Dis. 2009;6:583-91.

60. Tasara T, Stephan R. Cold stress tolerance of Listeria monocytogenes: a review of molecular adaptive mechanisms and food safety implications. J Food Prot. 2006;69:1473-84.

61. Azizoglu RO, Kathariou S. Temperature-dependent requirement for catalase in aerobic growth of Listeria monocytogenes F2365. Appl Environ Microbiol. 2010;76:6998-7003.

62. Edgar RC. MUSCLE: a multiple sequence alignment method with reduced time and space complexity. BMC Bioinform. 2004:5:113.

63. Hall BG. SNP-associations and phenotype predictions from hundreds of microbial genomes without genome alignments. PLoS One. 2014;9: e90490.

64. Clayton EM, Daly KM, Guinane CM, Hill C, Cotter PD, Ross PR. Atypical Listeria innocua strains possess an intact LIPI-3. BMC Microbiol. 2014;14.

65. Camejo A, Buchrieser C, Couve E, Carvalho F, Reis O, Ferreira P, Sousa S, Cossart P, Cabanes D. In vivo transcriptional profiling of Listeria monocytogenes and mutagenesis identify new virulence factors involved in infection. PLoS Pathog. 2009;5:e1000449. 
66. Cabanes D, Sousa S, Cebria A, Lecuit M, Portillo FG, Cossart P. Gp96 is a receptor for a novel Listeria monocytogenes virulence factor, Vip, a surface protein. EMBO J. 2005;24:2827-38.

67. Jacquet C, Doumith M, Gordon J, Martin P, Cossart P, Lecuit M. A molecular marker for evaluating the pathogenic potential of foodborne Listeria monocytogenes. J Infect Dis. 2004;189:2094-100.

68. Ratani SS, Siletzky RM, Dutta V, Yildirim S, Osborne JA, Lin W, Hitchins AD, Ward TJ, Kathariou S. Heavy metal and disinfectant resistance of Listeria monocytogenes from foods and food processing plants. Appl Environ Microbiol. 2012;78:6938-45.

69. Parsons C, Lee S, Jayeola V, Kathariou S. Characterization of a novel cadmium resistance determinant in Listeria monocytogenes. Appl Environ Microbiol. 2016

70. Lebrun M, Audurier A, Cossart P. Plasmid-borne cadmium resistance genes are similar to cadA and cadC of Staphylococcus aureus and are induced by cadmium. J Bacteriol. 1994;176:3040-8.

71. Bay DC, Rommens KL, Turner RJ. Small multidrug resistance proteins: a multidrug transporter family that continues to grow. Biochim Biophys Acta. 2008; 1778:1814-38.

72. Beceiro A, Tomas M, Bou G. Antimicrobial resistance and virulence: a successful or deleterious association in the bacterial world? Clin Microbiol Rev. 2013:26:185-230.

73. Pombinho R, Camejo A, Vieira A, Reis O, Carvalho F, Almeida MT, Pinheiro JC, Sousa S, Cabanes D. Listeria monocytogenes CadC regulates cadmium efflux and fine-tunes lipoprotein localization to escape the host immune response and promote infection. J Infect Dis. 2017:215:1468-79.

74. Lebrun M, Loulergue J, Chaslus-Dancla E, Audurier A. Plasmids in Listeria monocytogenes in relation to cadmium resistance. Appl Environ Microbiol. 1989;58:3183-6.

75. McLauchlin J, Hampton MD, Shah S, Threlfall EJ, Wieneke AA, Curtis GDW. Subtyping of Listeria monocytogenes on the basis of plasmid profiles and arsenic and cadmium susceptibility. J Appl Microbiol. 1997;83:381-8.

76. Chattopadhyay S, Weissman SJ, Minin VN, Russo TA, Dykhuizen DE, Sokurenko EV. High frequency of hotspot mutations in core genes of Escherichia coli due to short-term positive selection. Proc Natl Acad Sci. 2009;106:12412-7.

77. Camprubí-Font C, Lopez-Siles M, Ferrer-Guixeras M, Niubó-Carulla L, AbellàAmetller C, Garcia-Gil LJ, Martinez-Medina M. Comparative genomics reveals new single-nucleotide polymorphisms that can assist in identification of adherent-invasive Escherichia coli. Sci Rep. 2018;8:2695.

Ready to submit your research? Choose BMC and benefit from:

- fast, convenient online submission

- thorough peer review by experienced researchers in your field

- rapid publication on acceptance

- support for research data, including large and complex data types

- gold Open Access which fosters wider collaboration and increased citations

- maximum visibility for your research: over $100 \mathrm{M}$ website views per year

At BMC, research is always in progress.

Learn more biomedcentral.com/submissions 